\title{
Range estimates of distant visual stimuli*
}

\author{
EUGENE GALANTER and PATRICIA GALANTER \\ Columbia University, New York, New York 10027
}

\begin{abstract}
Os made magnitude estimations of the range of visual targets located at physical distances from a few hundred yards or less to more than 5 miles. The targets were at different elevations in different experiments so that $O$ 's gaze varied from 0 to $90 \mathrm{deg}$. The targets were presented against the empty sky or against water. The Os were stationary in most experiments, but were in motion in one. Results show that the psychophysical functions are power functions whose exponents range from ca. 1.25 to 0.8 , depending on the angle of the target above the horizontal. Background texture or $O$ motion had no effect.
\end{abstract}

People can obviously negotiate effectively in three-space plus time; they reach and lift, they run and catch, and often with great precision. However, just how information from the visual environment is processed to accomplish these tasks is not well understood. Principles of binocularity and cues and clues for three-dimensional perception are not really satisfactory as explanations of these performances. Indeed, the first questions about these space-related behaviors, viz, just what they are and how well they can be performed, have hardly been answered (Smith \& Smith, 1966). But the fact that people can move easily in a crowded room, and operate high-speed vehicles in which four-dimensional information must be processed rapidly, may not be the relevant target performance to be analyzed. First, we argue, we should understand what the psychophysical dimensions of such skills are. So, for example, we may ask whether there is a psychological representation of physical distance, and if there is, whether it is linear with physical space. If it is not linear, we then need to know whether this information is critical for performance of tasks involving translation in three dimensions. If it really is of importance in these tasks, we must recognize that such performances must include in their psychic organization a nonlinear transform. On the other hand, if such performance skills do not depend on the reduction of psychological representations of space and distance, but rather on other more easily processed variables, we may simply have wrongly attributed command and control activities to the psychological representations.

\section{EXPERIMENT I}

In 1964-66, we conducted experiments that showed that Os on the ground who were estimating the distance from their positions to aerial targets flying at low altitudes consistently overestimated those distances. This result is not unusual and has been found in past experiments (Applied Psychology Corporation, 1962; McCluskey et al, 1968). However, these previous results

*Research for this report was supported in part by the Engineering Psychology Programs, Office of Naval Research. simply reported the physical magnitudes of the overestimations that were observed when Os ranged aerial targets at a few fixed distances. Our efforts were directed toward mapping the entire psychophysical function to determine the form of the function that described the overestimation. Figure 1 shows data based on magnitude estimation scaling procedures used with two kinds of judgmental methods: a named standard with a modulus and no standard.

For this experiment, Os were located on a missile launch pad on a beach front overlooking the Pacific Ocean while an aircraft traversed a line perpendicular to their line of regard at various distances over many trials. The range of distances used was from 200 to

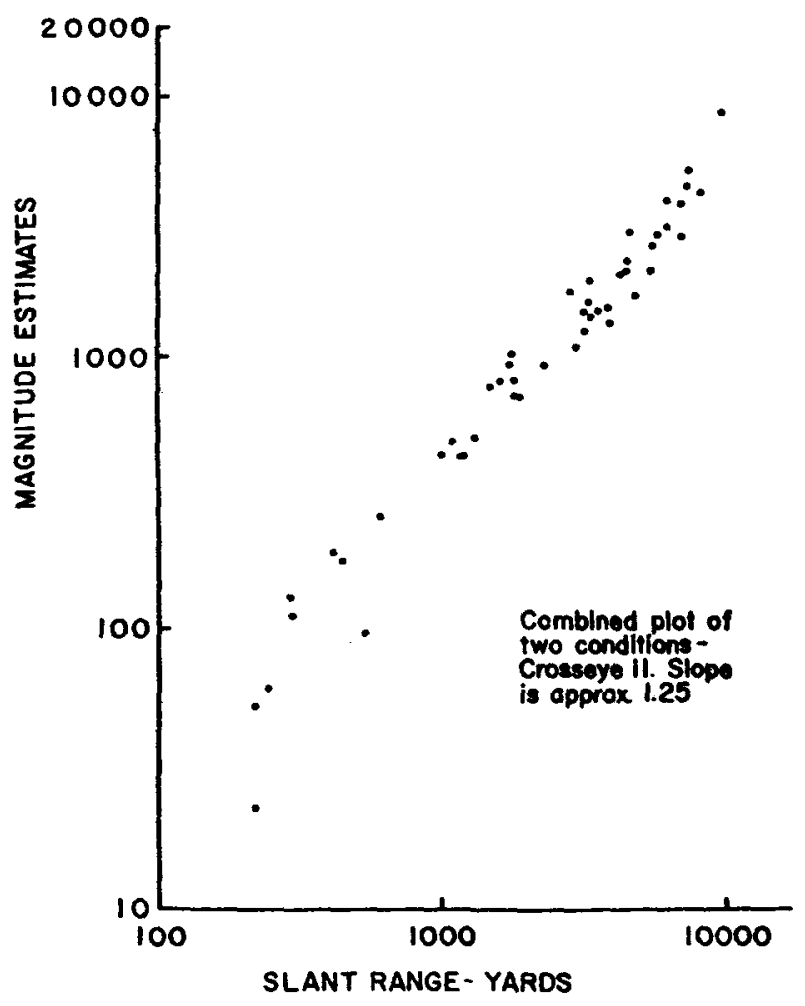

Fig. 1. Magnitude estimations of the perceived distance of a low-flying aircraft passing perpendicular to the Os' line of sight. The data points represent the medians of 12 judgments. 


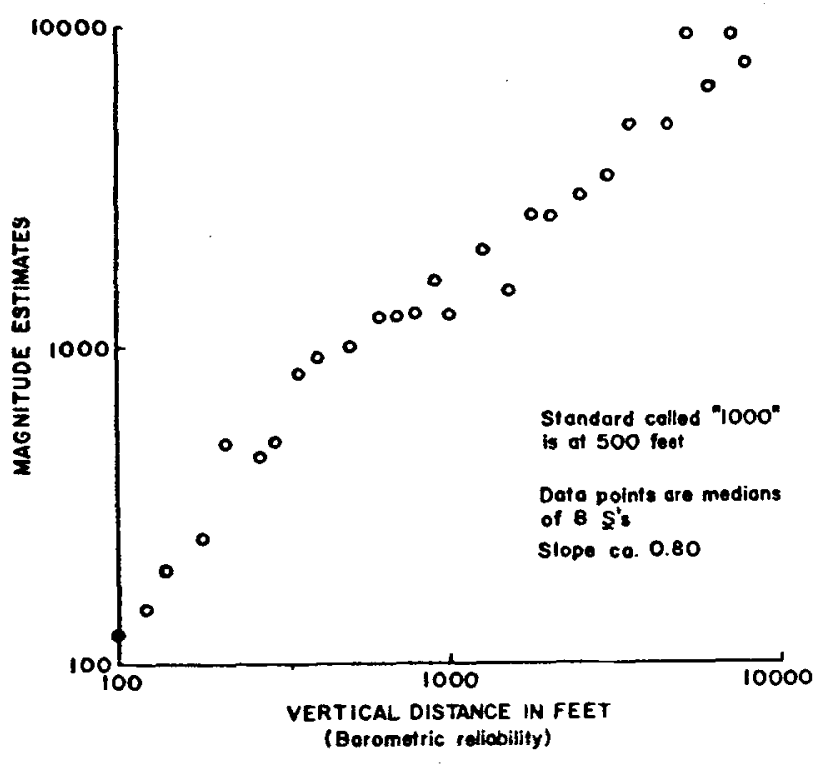

Fig. 2. Magnitude estimations of the perceived altitude of an aircraft passing over the Os' heads at varying vertical distances. Each data point is the median of 16 judgments.

9,861 yards. The order of the transits relative to their distance was irregular. The direction of the passes alternated from trial to trial. Twelve Os, who had been given $11 / 2 \mathrm{~h}$ of training in making magnitude estimations of line lengths, made magnitude estimation judgments of the distance of the aircraft on a signal from the range controller as the aircraft passed in front of them. The altitude of the aircraft was never greater than $200 \mathrm{ft}$ above the water.

Each data point in the psychophysical function shown in Fig. 1 represents the median of 12 judgments, one by each of the Os. The distance to the aircraft was determined by a digital radar (Model AN/FPS 16) and a real-time on-line computer, provided by the Pacific Missile Range, Point Mugu, California. ${ }^{1}$ The data points shown here in log-log coordinates are adequately described by a power function of the form

$$
\text { perceived distance }=(\text { actual distance })^{\mathbf{n}}
$$

The value of the exponent ( $\mathrm{n}$ ) for these data is 1.25 , and the exponent has been consistently reproduced under these and similar experimental conditions. Other researchers (Künnapas, 1960; Teghtsoonian \& Teghtsoonian, 1969) have obtained similar positively accelerated power functions.

\section{EXPERIMENT II}

Whereas all of the preceding data seem to hang together pretty well, certain phenomenological observations and controlled experiments have suggested that such results may be a function of the angle of regard, and may possibly be contingent on the nature of the intervening terrain (or lack thereof). The source of the first concern about angle of regard is the phenomenon known as the moon illusion. It has been consistently observed both by psychologists and others (Holway \& Boring, 1940) that the horizon moon appears much larger, perhaps by a factor of two, than the zenith moon. A variety of theories have been proposed to account for these observations (Kaufman \& Rock, 1962), the most reasonable of which is that the distance to the horizon appears to be greater than the distance to the zenith and there is some postulated size-distance relation; that is, that the shape of the sky is a flattened bowl rather than a hemisphere. If this conjecture is true, then the perceived distance to objects at the zenith should be different from what we have observed in our experiments and from what has been reported in the experiments of others. Consequently, we engaged upon further experiments to provide data to answer this question.

Eight Os (graduate students and technical personnel from the Psychophysics Laboratory at Columbia University) served in this experiment. All of them had had previous experience in making magnitude estimations of line lengths, and all of them understood the magnitude estimation procedure using a standard with a fixed modulus.

The target vehicle for the judgments was a light aircraft flown directly overhead at altitudes varying from 100 to $10,000 \mathrm{ft}$. The altitude for each pass was determined by a prearranged irregular schedule of altitudes. Os were told to call the first pass 1,000 . The aircraft for this pass traversed the O's station at $500 \mathrm{ft}$. On each subsequent pass, the $O$ s were required to estimate the altitude of the aircraft relative to the standard that they called 1,000 .

Figure 2 shows the psychophysical function relating magnitude estimations to the vertical distance of the target. Each data point represents two judgments by each of the eight Os for each altitude. Once again, a power function is a reasonable description of the data. However, the exponent for these data (with the first three points deleted), when determined by the method of least squares, is 0.8 . This value is clearly different from the value observed in our earlier experiments for horizontal lines of regard, and also departs remarkably from most other reported values. In this experiment, the Os consistently reported that the target was closer than it actually was. The unhappy choice of the modulus " 1,000 " against a physical standard of $500 \mathrm{ft}$ makes this feature of the data less obvious than it would otherwise be. Furthermore, the slope of the function is made to appear steeper than it is by the three lowest data points, which rise more sharply than the rest of the data. This result is common in magnitude estimation judgments in many modalities (Galanter \& Messick, 1961) and represents the source of the consistently reported need to modify the usual power function formula to include an additive constant. 


\section{EXPERIMENT III}

In order to determine whether the horizontal and vertical lines of regard are special and unique, another experiment was performed to obtain the psychophysical function of perceived distance against the physical range at an angle of regard somewhere between the horizontal and the vertical. In this experiment, 14 Os (enlisted seamen familiar with aircraft operations) were used to make the judgments. This time, as the aircraft made passes perpendicular to the line of regard at different distances, it was also at different elevations. The distances and altitudes were monitored by an instrument landing system radar sweep with the glide slope antenna angle toggled to $12 \mathrm{deg}$. The values of the physical variable of this experiment are less precise than they were in the previous study, but to compensate partially for this lack of precision in the physical variable, each of the Os made 10 judgments at each of the distances used. For this experiment, no fixed standard was used. This experiment was run on 4 different days because of the number of judgments used. There were slight variations in weather conditions on these days; however, days were chosen when weather conditions provided at least 7 miles of reported visibility, and the cloud cover was at most scattered at $5,000 \mathrm{ft}$ or higher.

Os made their judgments on command of the range commander when the aircraft was abeam the O's station. The range commander's "mark" was also transmitted to the radar operator, who recorded the range and the angular accuracy of the run. If the run departed from $12 \mathrm{deg}$ by more than the glide slope radar sweep excursion, the trial was discarded. Os used their own standards, and the data were reduced by multiplicative constants to minimize the overall variability of the judgments.

Once again, the results graphed in Fig. 3 can be described as a power function, albeit a degenerate one insofar as the slope has a value very near 1 . Thus, the distance judgments made by these Os in this experiment conform very well to the actual distances between them and the aircraft.

From these data, and the results of earlier experiments, we may safely conclude that the perceived distance of a target is (to a first approximation) a power function of the physical distance of the target. However, the two experiments reported above indicate that the parameter of the power function-the value of exponent-is contingent upon the angle of regard (or something correlated with the angle of regard) through angles ranging from the horizon $(0 \mathrm{deg})$ to the zenith (90 deg). The most reasonable conjecture to describe this parametric change is that the perceived distance at the horizon consistently overestimates the actual distance, the perceived distance at the zenith consistently underestimates the actual distance, and in between there is probably a smooth transition, with reduced overestimation as the angle of regard increases,

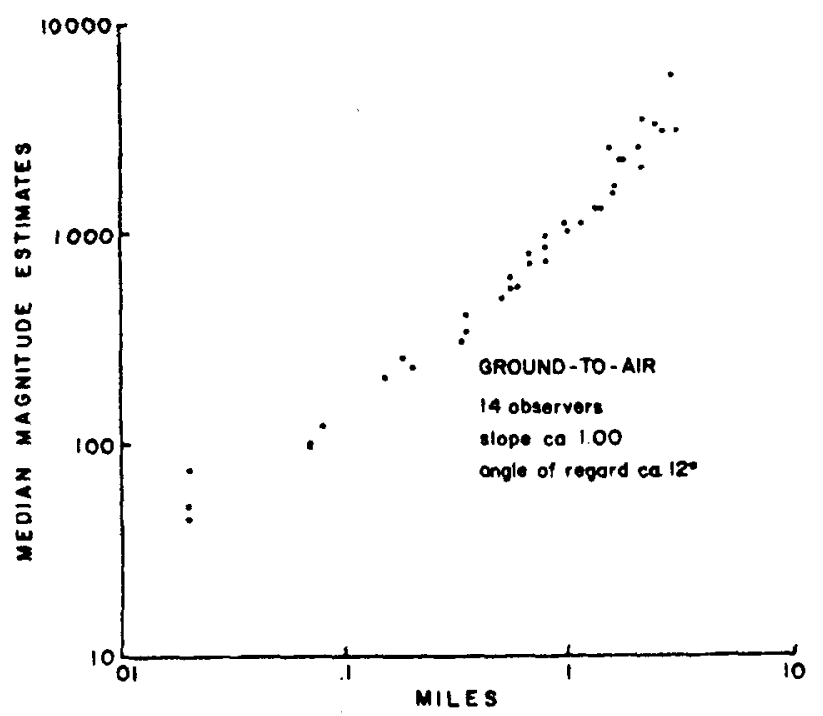

Fig. 3. Magnitude estimations of the perceived distance of an aircraft passing in front of the $O$ s at a constant angle of $12 \mathrm{deg}$ above the horizon. Each point represents the median of 140 judgments.

until finally underestimation begins and reaches its maximum at $90 \mathrm{deg}$. Such a labile distance function, contingent as it seems to be upon the angle of regard, does not speak well for it as the psychological determinant of complex psychomotor tasks.

However, it still remains to be seen whether this distance function may stabilize under certain sets of conditions. In particular, in the experiments described so far, the target is an aerial target, that is suspended or located against a background of indeterminate structure, the sky. Our next experiment was designed to find out whether the perceived distance function was altered if the target object was imbedded in an environment with some continuously graded microstructure. Although these conditions were found in the earliest studies of distance estimation, an exact comparison is difficult to make because of the methods of data reduction that were employed in the earlier studies. Consequently, Experiment IV was conducted to try to determine whether range estimations of maritime objects conform to the general law of the psychophysical function that we had observed for aerial targets.

\section{EXPERIMENT IV}

This experiment required that Os on the shore of a large bay estimate the range of a small boat passing on a line perpendicular to their line of regard at varying distances. For this experiment, Great Bay, New Jersey, was used, with a $26-\mathrm{ft}$ inboard cruiser serving as the target vehicle. A tall mast was erected on the aft end of the vessel to provide easy range finder ranging. The ranging of the vessel was accomplished with an ND4 


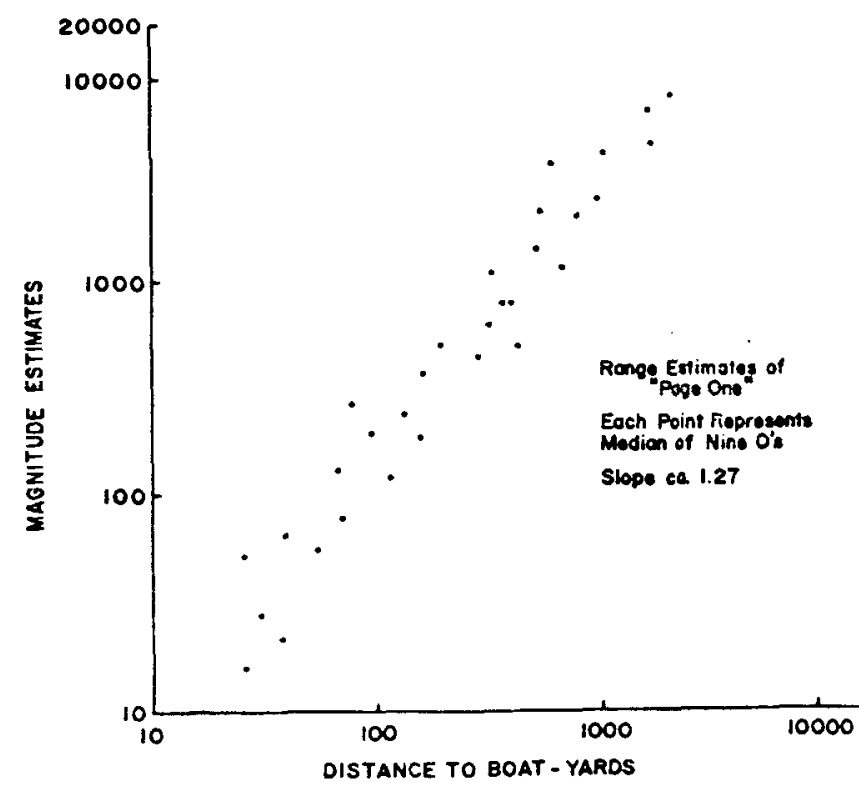

Fig. 4. Magnitude estimations of the perceived distance of a boat passing perpendicular to the line of sight of Os on the shore. Each point represents the median of nine judgments.

Mark 2 U.S. Navy range finger with a $1-\mathrm{m}$ base. The dynamic range of this range finder is 200 to 10,000 yards. At distances less than 200 yards, a small Edmund Scientific Corporation split-image range finder was used. This small range finder was calibrated up to $300 \mathrm{ft}$ with a surveying tape. During its runs, the boat maintained a constant compass course (or its reciprocal) but varied its distance from the observation point according to an irregular schedule of qualitative distances, e.g., very far, near, fairly near, etc.

Nine Os, employees from the National Aviation Facilities Experimental Center at Atlantic City volunteered to make the observations. They were all given $30 \mathrm{~min}$ of instruction in magnitude estimation methods with line lengths as stimuli. They were instructed to make their judgments without an E-determined standard. The vessel was in communication with the range commander by radio. On each run, the range commander called "mark" as the vessel passed abeam of the Os. This designated that a judgment was to be made. The angle of regard was essentially horizontal. The range of the vessel dring its transit was taken by an assistant at the observation post and recorded at the call of "mark." If the range could not be determined at the "mark," the assistant reported a mistrial and the results were struck from the data records. Os completed a total of 31 observations. Median values were determined and the results graphed in Fig. 4. Observe that the slope of the function is approximately 1.27, quite close to the observed function for low-angle aircraft. It need hardly be added that the function itself is a power function, as is found in all of our previous studies. We draw the conclusion that imbedding the vehicle in a background with a texture gradient-water-in no way changes the nature or value of the psychophysical judgments of distance.

\section{EXPERIMENT V}

The previous results tend to show that the psychophysical function relating physical distance to perceived range is a power function whose exponent depends, not on variables such as atmospheric transmissivity or background texture, but rather on angle of regard, insofar as Experiment IV varied the texture without changing the exponent, and results under varying sky conditions were similar. However, there is a specific feature of all the previous experiments that may be relevant, and that is that the $O$ is stationary. The normal form of human interaction with environmental events involves movement of the person. Perhaps the static $\mathrm{O}$ in the preceding experiments induces the nonlinearity in the psychophysical functions. The moving $\mathrm{O}$ may transmute the judgments that would be made under static conditions in such a way as to reveal to us linear percepts on which the control of his activities may depend. Additionally, in the experiments reported above, the angles of regard ranged over, at most, $100 \mathrm{deg}$. We did no ranging from high vantage points. Therefore, to round out our understanding of these phenomena, we decided to examine the perceived distance function when the $O$ was undergoing dynamic translations and the targets remained fixed in the perceptual sense of that term. That is to say, the distal targets in the perceptual field were stationary relative to the surface of the earth.

In the first part of this experiment, six Os, untrained and unsophisticated ground personnel at the Naval Air Station, Lakehurst, New Jersey, who had no experience in light aircraft, served as Os. They worked in groups of three. Three of the Os would board the experimental aircraft, and it would fly at altitudes between 200 and $500 \mathrm{ft}$ over a prearranged course. As the aircraft crossed a known landmark, the Os were required to range an easily identifiable target on the ground. The Os had been given experience in the magnitude estimation of line lengths, and they did the ranging with no standard established by the $\mathrm{E}$.

The physical distances between the landmarks that were overf $\cdots, n$ and the targets that were ranged by the Os were determined by a combination of electronic, photogrammetric, and topographical survey techniques. Each target was physically measured from the overflown landmark by at least two independent metric procedures. Several times in the course of the experiment, Os became airsick because of the uncomfortable turbulence at low altitude. When this occurred, the runs were cut short and the aircraft returned to home base. Repeated experimental sessions were conducted on days when weather conditions were as similar as possible. The weather during the experiment 
was clear, with visibility of 7 miles. The temperature in the aircraft often reached above $90 \mathrm{deg}$. and consequently the conditions of the experiment for the Os would not normally be expected to lead to high-quality data.

Figure 5 plots the results obtained in this experiment from the six Os. The impressive consistency and regularity of the data speak well of the low variability with which the physical metric was established. The surprising result is the magnitude of the power function exponent, ca. 1.27. This is almost identical to ground-to-air estimates at horizontal lines of regard. That the angle of regard was fairly flat can be seen by the conditions of the observations. The average altitude of the aircraft was ca. $350 \mathrm{ft}$. The closest objects that were ranged were of the order of 250 yards, with the great bulk of data having been collected between 1 and 4 miles. It can be concluded that the angle of regard was rather low.

In order to assess the reliability of the $O s$ in this difficult task, a single well-practiced $E$ served as an $O$, and made repeated observations of each target. Figure 6 shows the results of 43 observations per data point, with an estimate of the variability of the physical metric given by the length of the horizontal line. For this single trained $O$, it is evident that the slope function, although slightly flattened at 1.2 , approximates what was observed for the untrained group of Ss.

We have now observed in a variety of experimental contexts that the judgments of visual distance into the third dimension by both practiced and unpracticed Os

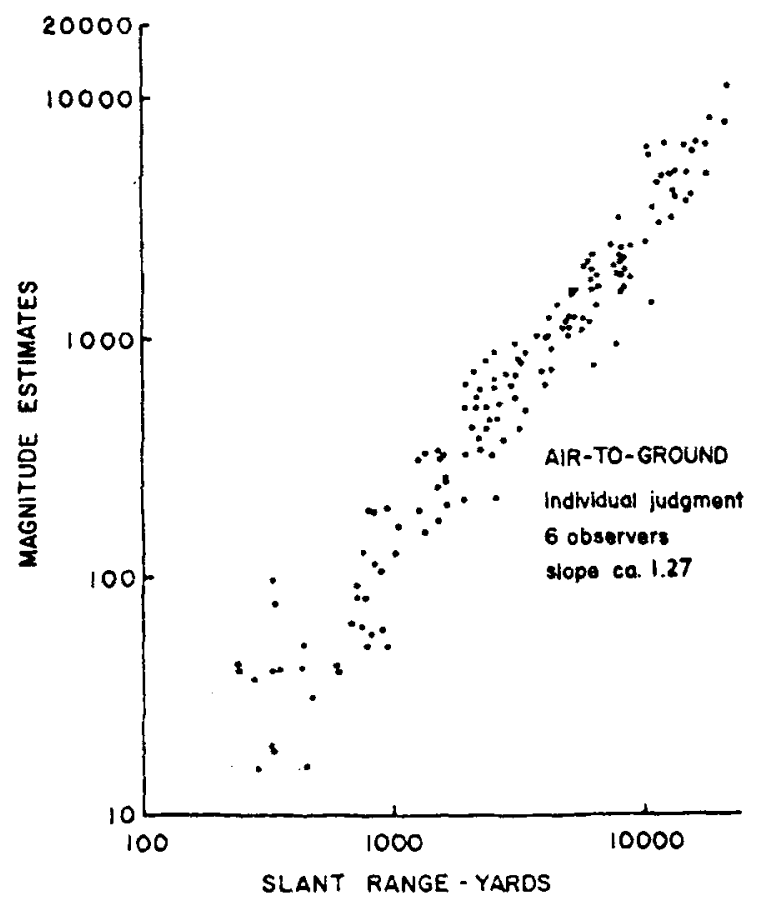

Fig. 5. Magnitude estimation of the perceived distance between $O s$ in an airplane and targets on the ground. Each point represents the median of six observations.

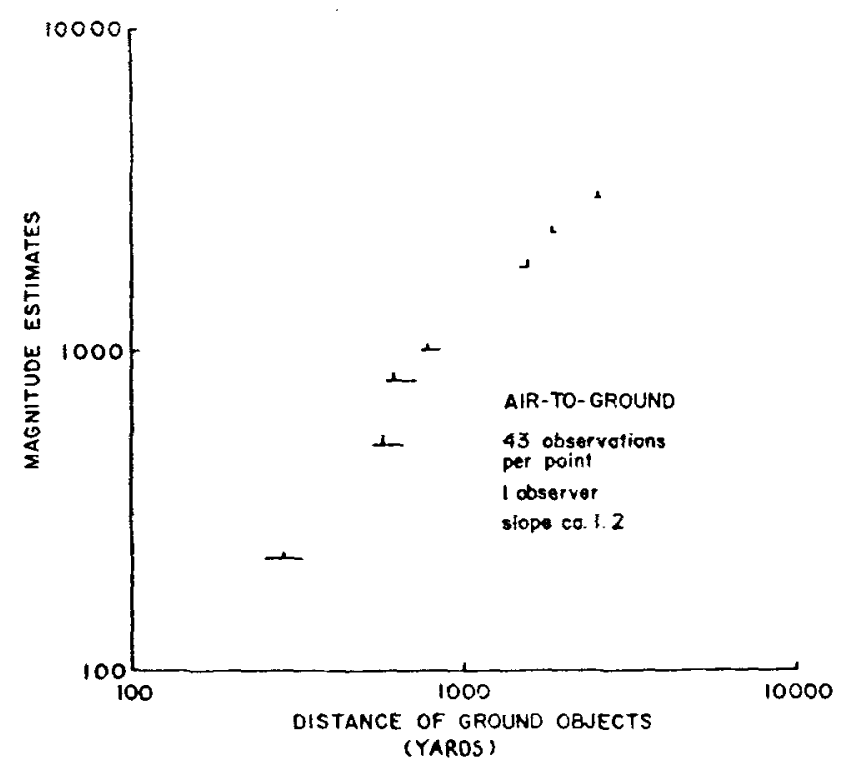

Fig. 6. Repeated magnitude estimations of the distance of ground targets obtained from a single $O$ in an airplane. Each data point represents the median of $\mathbf{4 3}$ observations.

consistently generate nonlinear psychophysical functions. If the perceptual information on which many kinds of human action are based is contingent upon perceptual data of the form that we have observed, then the control of such action must entail nonlinear components. This, of course, is no high crime, except that it certainly complicates any attempt to explicate the nature of perceptual motor organization.

\section{DISCUSSION}

Our data for horizontal lines of regard conform to past data collected indoors. The situation is more complicated when these data are compared to judgments that were made out-of-doors. For example, Teghtsoonian and Teghtsoonian (1970) report power functions as the form of the perceived distance function, but the exponents range from 0.85 to 0.99 for horizontal viewing. Of course, their ranges are small in comparison to those reported here, but if anything, their data suggest that the slope flattens as the range increases.

However, unlike the present experiment, this most comparable study used stimuli whose sizes varied by a factor of two. The different sized stimuli were used irregularly at the varying distances. Here, on the other hand, our stimulus was constant in size and fairly constant in velocity. A fine-grained analysis of the Os' viewing time and the velocity of the target for Experiment I showed that they used a fairly constant viewing time, ca. $3.5 \mathrm{sec}$. and that the vehicle maintained velocity to within $7.6 \%$. One way to minimize the incompatibility of our results and those of the Teghtsoonians is to conjecture that judgments of distance are always contingent on the size-distance correlation at the proximal stimuli. The reduction of this 
correlation in the Teghtsoonians' experiments may be the basis for the observed difference in the slope functions between the two sets of experiments.

Although various aspects of the field research described in the preceding pages are still in progress, we can already draw some tentative conclusions based on the data shown here and on the existing literature. First of all, naturalistic observation guarantees that normal people learn to negotiate through very complex close visual environments without injuring themselves or others in the first dozen years of their lives. The precision with which this type of performance occurs, and its probable extension to similar behavior that involves the guidance of high-speed vehicles through distant visual environments later in the person's life, leads to questions about how this behavior can be guided and to questions about the mechanisms by which environmental information is processed for use in such guidance. The first and most obvious answer is that the appearance of the world as perceived by the person guides his actions. What the preceding experiments suggest in conformity with a varied experimental literature (Hochberg, 1971, p. 513ff) is that the appearance of the world as measured here may have metric properties that are not linearly related to the metric properties of the physical world itself. This implies that some nonlinear transformation of the psychological information would have to take place if the person's behavior was expected to conform with the realities of the physical world. It is not impossible that such a transformation occurs; however, such nonlinearity certainly suggests that alternative hypotheses to explain the guidance of skilled performance are not out of order.

The currently accepted view of motor skills, tracking, and sensory motor performance is still the response shadowing concept first introduced in the early part of the second world war to serve as a basis for analysis of flexible gunnery and other pursuit-type tasks. These theories all presume that stimulus information guides responses with linear fidelity, except for a remnant attributable to error factors (Bilodeau \& Bilodeau, 1969). The alternative characterization that we suggest as an account of human performance and guidance in dynamic situations is a variant of the proposal suggested by Miller, Galanter, and Pribram (1960). Such a model interprets components of the skilled performance as "psychologically" ballistic. The model requires only that after the completion of some act it must be possible for the person to check the results of his act against internally stored perceptually based information. His task is now to determine whether new information, arriving as a consequence of the ballistic act itself, conforms to the expectations based on the stored information. In the context of such a model, nonlinearities in the actual perceptual structure of the environment would have no complicating consequences. The nearest analysis of human performance that contains similar ideas is that of Schmidt (1968), who limited his attention to the study of temporal prediction. We shall have more to say on this topic at a later date. Some early results on this topic are reported in Galanter (1972). ${ }^{2}$

\section{REFERENCES}

Applied Psychology Corporation. Pilot judgments of aircraft range and relative altitude: Ground-to-air and air-to-air observations. Technical Reports 10 and 11, Applied Psychology Corporation, Arlington, Virginia, June 1962.

Bilodeau, E. W., \& Bilodeau, I. M. (Eds.) Principles of skill acquisition. New York: Academic Press, 1969.

Balanter, E., \& Messick, S. The relations between category and magnitude scales of loudness. Psychological Review, 1961, 68, 363-372.

Galanter, E., \& Messick, S. The relations between category and magnitude scales of loudness. Psychological Review, 1961, 68, 363-372.

Galanter, E. Range and time estimates of dynamic visual targets. Technical Report 27, Psychophysics Laboratory, Columbia University, 1972.

Hochberg, 3. Perception. II: Space and movement. In J. W. Kling and L. A. Riggs (Eds.), Woodworth \& Schlosberg's Experimental psychology. (3rd ed.) New York: Holt, Rinehart $\&$ Winston, 1971. Chap. 13.

Holway, A. F., \& Boring, E. G. The moon illusion and the angle of regard. American Journal of Psychology, 1940, 53, 109-116.

Kaufman, L., \& Rock, I. The moon illusion. Scientific American, $1962,207,120-130$.

Künnapas, T. Scales for subjective distance. Scandinavian Journal of Psychology, 1960, 1, 187-192.

McCluskey, M. R., Wright, A. D., \& Frederickson, E. W. Studies on training ground observers to estimate range to aerial targets. Technical Report 68-5, HumRRO, May 1968.

Miller, G. A., Galanter, E., \& Pribram, K. Plans and the structure of behavior. New York: Holt, 1960.

Schmidt, R. A. Anticipation and timing in human motor performances. Psychological Bulletin, 1968, 70, 631-646.

Smith, O., \& Smith, P. G. Developmental studies of spatial judgments by children and adults. Perceptual \& Motor Skills, 1966, 22, 3-73.

Teghtsoonian, M., \& Teghtsoonian, R. Scaling apparent distances in natural indoor settings. Psychonomic Science, 1969, 16, 281-283.

Teghtsoonian, R., \& Teghtsoonian, M. Scaling apparent distances in a natural outdoor setting. Psychonomic Science, 1970, 21 , 215-216.

\section{NOTES}

1. We extend our sincere thanks to Robert Maples and Ray Morton, range operation officers at the Pacific Missile Range for their invaluable assistance in the conduct of this research.

2. In various of our early experiments, we were aided by George Semb. In the later work, the aid of John A. Owens is acknowledged. Professor Michael Kahn helped plan the experimental procedures and flew the first missions of our unreported shake-down experiments in 1964.

(Received for publication March 12, 1973; revision received April 19, 1973.) 